\title{
Fatores associados a não realização de Papanicolau em mulheres quilombolas
}

\author{
Factors associated with failure to take a Pap smear test \\ among Quilombola women
}

Márcio Vasconcelos Oliveira ${ }^{1}$

Mark Drew Crosland Guimarães ${ }^{2}$

Elisabeth Barboza França ${ }^{2}$

${ }^{1}$ Faculdade de Farmácia, Instituto Multidisciplinar em Saúde, Universidade Federal da Bahia-campus Anísio Teixeira. R. Rio de Contas 58/Quadra 17/Lote 58, Candeias. 45029-094 Vitória da Conquista Bahia Brasil. marciomvo@ig.com.br ${ }^{2}$ Programa de PósGraduação em Saúde Pública e Grupo de Pesquisas em Epidemiologia e Avaliação em Saúde (GPEAS), Faculdade de Medicina da Universidade Federal de Minas Gerais.
Abstract The objective of this study was to analyze factors associated with failure to take a Pap smear test among quilombola women living in Vitória da Conquista in the state of Bahia. A cross-sectional, population-based study was conducted with women aged 18 to 64. Descriptive analysis and univariate and multivariate analysis using a multinomial logistic model was applied. Women who had never performed the test or had not had one for over three years were compared separately from those who were examined within the preceding three years. Of the 348 women included in the analysis, $27.3 \%$, reported never having a Pap smear test. The following factors were independently associated with never having taken the test: age of 18 to 29 years and 50 to 59 years; lack of education; not having a partner; seeking care in health-related units/establishments other than their place of residence; and having a clinical breast examination three or more years ago or never having one. The findings indicate a need for reflection in order to combat factors that are associated with not having a Pap smear test among quilombola women, since it is important to implement actions for the prevention of cervical cancer. Key words Pap smear test, Cross-sectional studies, Cervical cancer, Quilombolas, Prevention
Resumo O objetivo deste estudo foi analisar os fatores associados a não realização do exame de Papanicolaou entre as mulheres quilombolas residentes em Vitória da Conquista, Bahia. Foi utilizado delineamento de estudo transversal de base populacional, com inclusão de mulheres com idade de 18-64 anos. Foram realizadas análise descritiva e de associação univariada e multivariada utilizando modelo logístico multinomial. Mulheres que nunca realizaram o exame ou o realizaram há mais de três anos foram comparadas separadamente com aquelas que realizaram o exame há menos de três anos. De 348 mulheres incluídas na análise, 27,3\% afirmaram nunca ter realizado o Papanicolaou. Foram associados de forma independente com nunca ter feito o exame: faixa etária de 18 a 29 anos e 50 a 59 anos, não ter instrução, não ter companheiro, buscar atendimento relacionado à saúde em unidades/estabelecimentos diferentes de seu local de residência e ter realizado exame clínico das mamas há três ou mais anos ou nunca tê-lo realizado. Os achados indicam uma necessidade de reflexão, com o objetivo de melhor enfrentamento dos fatores que se associam à não realização do exame Papanicolaou entre as mulheres quilombolas, sendo importante contemplar ações de prevenção para o câncer de colo uterino.

Palavras-chave Exame de Papanicolaou, Estudos transversais, Câncer de colo do útero, Quilombolas, Prevenção 


\section{Introdução}

O câncer de colo uterino (CCU) é uma doença de grande relevância mundial e chega a ser a mais comum nas mulheres em algumas regiões do mundo como África, Ásia e América Central. Taxas de incidência superiores a 30 por 100.000 mulheres ocorrem, resultando em aproximadamente 500 mil casos novos por ano no mundo. Apenas em 2010, o CCU foi responsável por mais de 200 mil mortes no mundo, a maioria tendo ocorrido em países menos desenvolvidos ${ }^{1}$. No Brasil, representa o segundo tumor mais frequente entre as mulheres, com exceção dos não melanoma da pele. Foram estimados $17.540 \mathrm{ca}-$ sos novos de CCU em 2012, correspondendo a uma taxa de 17 casos por 100 mil mulheres sendo que, para a Bahia, estimou-se uma taxa de 13,5 casos/100 mil mulheres ${ }^{2}$.

O câncer de colo de útero ocorre em decorrência da infecção pelo Papilomavírus Humano (HPV), apesar de ser esta uma condição necessária, mas não suficiente. Outros fatores de risco tais como a idade (45-49 anos), início precoce da atividade sexual, multiplicidade de parceiros sexuais, tabagismo, baixa condição socioeconômica, são importantes ${ }^{3-6}$.

O CCU é uma doença de crescimento lento e silencioso, com uma fase pré-clínica em que a detecção de possíveis lesões pré-malignas precursoras pode ser feita por meio da realização periódica do exame de Papanicolaou ${ }^{4}$, um exame seguro, barato, eficaz, de fácil execução e aceitação pela população feminina ${ }^{7-9}$. Uma boa rastreabilidade citológica atrelada ao tratamento adequado nos estágios lesivos iniciais pode resultar em uma redução de até $90 \%$ das taxas de incidência de câncer de colo uterino, quando o rastreamento apresenta boa cobertura populacional $(80 \%)$ e é realizado dentro dos padrões de qualidade ${ }^{10-13}$.

No Brasil, as diretrizes para o rastreamento do câncer de colo do útero recomendam a realização do exame citopatológico anualmente em mulheres com faixa etária entre 25 e 64 anos, com intervalo de três anos após dois exames consecutivos negativos. No entanto não pode ser ignorada a triagem nos mesmos moldes entre as mulheres sexualmente ativas com idade inferior a 25 anos, devido à etiologia do CCU.

Desde a década de 90, o Ministério da Saúde (MS), valendo-se de diretrizes do Instituto $\mathrm{Na}$ cional do Câncer (Inca), vem elevando a cobertura de exames preventivos para $\mathrm{CCU}$ em números absolutos.
A partir de 1998, houve uma significativa e crescente ampliação da oferta de exames citopatológicos no país ${ }^{14}$. Apesar destes esforços crescentes para melhorar a eficiência dos programas de prevenção, é persistente a manutenção de altas taxas de incidência e de mortalidade de CCU no Brasil, o que indica que as medidas adotadas podem não ter conduzido aos resultados espera$\operatorname{dos}^{14}$. É possível também que falhas no programa de rastreamento para CCU no Brasil contribuam para esta situação.

Ao analisar este programa em regiões e municípios brasileiros, constatam-se grandes discrepâncias com relação à sua cobertura e efetividade, provavelmente relacionadas à baixa disponibilidade de médicos e enfermeiros na rede de atenção básica e de laboratórios de citopatologia devidamente equipados para atender a população. Outra questão importante dentro deste contexto é a percepção da existência de limitações de acesso aos serviços de saúde por barreiras socioeconômicas, culturais e geográficas, sendo um problema a ser enfrentado pelos gestores do programa de controle do câncer de colo de útero ${ }^{4}$. Também são conhecidos e amplamente apontados na literatura diversos fatores associados a não realização do exame de Papanicolaou, como a idade avançada, o baixo nível socioeconômico, pertencimento a certos grupos étnicos como afrodescendentes, não ter cônjuge (solteiras, separadas e viúvas) ${ }^{15}$. $\mathrm{O}$ reconhecimento desses fatores como barreiras para a realização do exame preventivo é parte fundamental no enfrentamento a esta problemática.

No Brasil, a população negra experimenta maiores taxas de mortalidade por câncer se comparada à população geral, sendo provavelmente um dos reflexos de desigualdades nos âmbitos social, econômico, político e na saúde. Essa população traz consigo experiências desiguais ao nascer, viver, adoecer e morrer ${ }^{16}$. Isso pode ser observado pela má qualidade de vida, pobreza, baixa escolaridade, dificuldade de acesso aos serviços em geral, taxas de morbimortalidade mais altas do que as registradas em nível nacional, fome, desnutrição, riscos ocupacionais e violência social ${ }^{17}$.

As comunidades quilombolas se inserem dentro desse contexto, pois são de presumida ancestralidade negra e também experimentam situação de importante vulnerabilidade social, decorrente de um processo histórico de expropriação de cultura e de direitos, cujo impacto tem reflexo nos indicadores de saúde desta população $0^{14}$.

Assim, considerando que a realização do exame Papanicolaou está associada dentre outras 


\section{Métodos}

Os dados utilizados para este trabalho foram extraídos do Projeto COMQUISTA ${ }^{19}$, um estudo de corte transversal, de base populacional, realizado entre os meses de setembro e outubro de 2011 em comunidades quilombolas de Vitória da Conquista, Bahia. As comunidades quilombolas dispõem de atendimento por equipe multidisciplinar de saúde da família em caráter semanal ou quinzenal e atendimento diário por agente comunitário de saúde.

O município é atendido por 38 equipes, sendo 15 na zona rural. A rede de atenção à saúde municipal disponível é composta por 394 estabelecimentos, sendo 85 públicos, quatro filantrópicos e 305 de caráter privado, e está estruturada e hierarquizada em níveis de atenção primária, secundária e terciária. As necessidades dos usuários, envolvendo exames laboratoriais, diagnóstico de imagem, especialidades médicas, urgência e emergência, além de internações, levam os indivíduos à rede referenciada, atividade esta mediada e regulada pela central de marcação de consultas, procedimentos e exames. Existe uma cota mensal pré-definida de consultas, exames e procedimentos especializados para cada ESF e também para as secretarias municipais de saúde de outros 62 municípios pactuados, tendo em vista a atuação de Vitória da Conquista como polo macrorregional do sudoeste da Bahia ${ }^{20}$.

No projeto COMQUISTA, o plano amostral foi obtido através de estimativa populacional baseada nos dados disponíveis da Secretaria $\mathrm{Mu}$ nicipal da Saúde. O universo amostral estimado foi de 2.935 indivíduos adultos oriundos de 10 comunidades quilombolas, sediadas em cinco distritos do município. Para o cálculo do tamanho da amostra, foi considerada uma prevalência estimada de $50 \%$, dada a heterogeneidade dos eventos mensurados, precisão de $5 \%$, intervalo de confiança de $95 \%$, efeito de desenho de 2 e $30 \%$ de possíveis perdas, totalizando uma amostra final de 884 indivíduos adultos. Registrou-se uma

proporção de perdas de 9,9\%, das quais 77,4\% ocorreram no sexo masculino. Ao final, foram realizadas 797 entrevistas, sendo 54,3\% $(n=433)$ de mulheres. $\mathrm{O}$ inquérito foi feito utilizando um questionário semiestruturado dividido em módulos, adaptado da Pesquisa Nacional de Saúde $(\mathrm{PNS})^{21}$, onde um grupo de profissionais da Saúde Pública avaliou cada questão separadamente, considerando o contexto a ser aplicado. Foram realizados dois pré-testes no intuito de verificar adequações de linguagem, sequência e seleção das perguntas rigorosamente necessárias.

As entrevistas foram realizadas nos domicílios por meio de computadores portáteis (HP Pocket $\mathrm{Rx} 5710)^{22}$. A investigação partiu de questões norteadoras existentes no módulo F (Saúde da mulher) do questionário, que possui um conjunto de perguntas relacionadas à saúde feminina, e seguiu as recomendações normatizadas pelo Inca para triagem com o exame de Papanicolaou. Foram elegíveis todas as mulheres com idade de 25 a 64 anos, independentemente de relato de ter tido ou não atividade sexual, e aquelas com idade de 18 a 24 anos que relataram ser sexualmente ativas. Segundo estes critérios, 348 mulheres foram selecionadas.

Neste estudo, considerou-se a realização do exame Papanicolau como variável dependente, que foi assim categorizada: (0) mulheres com realização de Papanicolaou há menos de três anos; (1) mulheres que realizaram o exame com três ou mais anos; e, (2) aquelas que nunca realizaram. As mulheres quilombolas que nunca realizaram Papanicolaou e aquelas que realizaram há três ou mais anos foram comparadas com as que realizaram o exame há menos de três anos, padrão recomendado. Foram consideradas como possíveis variáveis explicativas: a) variáveis sociodemográficas: faixa etária, renda, escolaridade, estado conjugal; b) variável representativa do estado de saúde: autoavaliação do estado de saúde, c) variáveis marcadoras de acesso aos serviços de saúde: consulta médica nos últimos 12 meses e unidade/local de busca para atendimento relacionado à saúde (se mesmo ou diferentes); d) variáveis marcadoras de utilização de serviços de saúde: realização de exames preventivos para câncer de mama: (exame clínico das mamas [ECM] e mamografia), dosagem de glicose, dosagem de colesterol/triglicerídeos e verificação da pressão arterial; e) variáveis relacionadas ao estilo de vida: prática de atividade física nos últimos três meses, consumo de álcool e tabagismo.

Foi realizada análise descritiva das variáveis com o banco de dados constituído em Microsoft 
Access Database (MDB) e com as análises realizadas através do programa estatístico EPI INFO (versões 3.5.1 e 7.0.9.7). Para a comparação de frequências foi utilizado o teste do qui-quadrado, adotando nível de significância de 5\%.

Procedeu-se em seguida às análises univariada e multivariada por meio de modelo logístico multinomial, tendo como grupo de referência as mulheres com realização do exame Papanicolaou há menos de três anos. Na modelagem, foram consideradas as variáveis que apresentaram valor $\mathrm{p}<0.20$ na análise univariada para inserção no modelo, e a variável renda, dada sua importância epidemiológica. No modelo multivariado final foi considerado o índice de significância de $\mathrm{p}<$ 0.05 . Para as análises foram utilizados o pacote estatístico EPI INFO (versões 3.5.1 3 7.0.9.7) para a univariada, e o R versão 2.11 para a multivaria- da. O modelo estatístico final foi avaliado pelos testes-Akaike's Information Criterion (AIC) e pela análise da Deviance residual, que indicaram boa adequação.

O projeto COMQUISTA foi aprovado pelo Comitê de Ética em Pesquisa da Faculdade São Francisco de Barreiras e pelo Comitê de Ética em Pesquisa da Universidade Federal de Minas Gerais.

\section{Resultados}

A Tabela 1 apresenta a análise descritiva da população de mulheres incluída neste estudo $(\mathrm{n}=$ 348). Verificou-se uma média de idade de 37 anos, renda per capita média de cerca de $\mathrm{R} \$ 199,00$ com aproximadamente $75 \%$ das mulheres tendo como renda menos de meio salário mínimo, que

Tabela 1. Análise descritiva da amostra de mulheres quilombolas participantes do estudo $(\mathrm{n}=348)$. Projeto COMQUISTA, Bahia, Brasil, 2011.

\begin{tabular}{|c|c|c|c|c|c|}
\hline & $\mathbf{n}$ & $(\%)$ & & $\mathbf{n}$ & $(\%)$ \\
\hline Faixa etária & & & Procura de atendimento relacionado à saúde & & \\
\hline $18-24$ anos & 70 & 20,1 & Mesmo local & 274 & 79,0 \\
\hline $25-29$ anos & 45 & 12,9 & Locais diferentes & 73 & 21,0 \\
\hline $30-39$ anos & 96 & 27,6 & Realização de mamografia & & \\
\hline $40-49$ anos & 74 & 21,3 & $<2$ anos & 19 & 5,5 \\
\hline $50-59$ anos & 47 & 13,5 & $\geq 2$ anos & 26 & 7,5 \\
\hline $60-64$ anos & 16 & 4,6 & Nunca realizada & 303 & 87,0 \\
\hline Escolaridade $^{\mathrm{a}}$ & & & Consumo de álcool & & \\
\hline Sem instrução & 77 & 22,4 & Não consome & 240 & 69,0 \\
\hline 1 - 4 anos de estudo & 162 & 47,1 & Consome $<1 \mathrm{vez} / \mathrm{mês}$ & 62 & 17,8 \\
\hline $5+$ anos de estudo & 105 & 30,5 & Consome $1+$ vezes/mês & 46 & 13,2 \\
\hline Estado conjugal & & & Tabagismo & & \\
\hline Sem companheiro & 117 & 33,6 & Não fumante & 314 & 90,2 \\
\hline Com companheiro & 231 & 66,4 & Fuma menos que diariamente & 12 & 3,5 \\
\hline Autoavaliação do estado de saúde ${ }^{*}$ & & & Fuma diariamente & 22 & 6,3 \\
\hline Boa & 153 & 44,1 & Última verificação de pressão arterial ${ }^{c}$ & & \\
\hline Regular & 159 & 45,8 & $<1$ ano & 269 & 78,0 \\
\hline Ruim & 35 & 10,1 & $\geq 1$ ano & 63 & 18,2 \\
\hline Renda $^{\mathrm{b}}$ & & & Nunca verificada & 13 & 3,8 \\
\hline$<1 / 2$ salário mínimo & 260 & 74,7 & Prática de atividade física (últimos 3 meses) & & \\
\hline$\geq 1 / 2$ salário mínimo & 88 & 25,3 & Sim & 60 & 17,2 \\
\hline Realização de Papanicolaou & & & Não & 288 & 82,8 \\
\hline$<3$ anos & 196 & 56,3 & Última realização de glicemia $^{* *}$ & & \\
\hline$\geq 3$ anos & 57 & 16,4 & $<2$ anos & 164 & 47,8 \\
\hline Nunca realizou & 95 & 27,3 & $\geq 2$ anos & 74 & 21,6 \\
\hline Realização do exame clínico das mamas & & & Nunca realizada & 105 & 30,6 \\
\hline$<3$ anos & 54 & 15,5 & Última dosagem de colesterol/triglicérides $^{* * *}$ & & \\
\hline$\geq 3$ anos & 30 & 8,6 & $<2$ anos & 134 & 39,4 \\
\hline Nunca realizou & 264 & 75,9 & $\geq 2$ anos & 54 & 15,9 \\
\hline Consultou médico nos últimos 12 meses & & & Nunca realizada & 152 & 44,7 \\
\hline Sim & 196 & 56,3 & & & \\
\hline Não & 152 & 43,7 & & & \\
\hline
\end{tabular}

a 4 não respondentes; ${ }^{\text {b }}$ salário equivalente a R $\$ 545,00 ;$ ‘ 3 não respondentes; 1 não respondente; ${ }^{* *} 5$ não respondentes; ${ }^{* * *} 8$ não respondentes. 
na época da pesquisa era de $\mathrm{R} \$ 545,00$. A maioria das mulheres tinha companheiro e $22,4 \%$ delas não tinham nenhuma escolaridade. A média de escolaridade encontrada foi de 3,6 anos de estudo. A maioria das mulheres autoavaliou seu estado de saúde como bom ou regular, relataram ser não fumantes e 56,3\% declararam ter procurado um médico nos últimos 12 meses. Entretanto, em geral, não praticaram atividade física nos últimos três meses e nunca realizaram exames preventivos para câncer de mama.

A prevalência de não realização do exame de Papanicolaou entre as mulheres foi de $27,3 \%$ (IC 95\%: 1,4 - 1,9). Chama atenção que, dentre essas,
$45,3 \%(\mathrm{n}=43)$ tinham idade entre 18 e 24 anos e afirmaram ser sexualmente ativas. A maioria não possuía companheiro, autoavaliou seu estado de saúde como bom, revelou ter renda menor que $1 / 2$ salário mínimo e escolaridade de cinco ou mais anos de estudo. A prevalência de realização do Papanicolaou há três ou mais anos foi de 16,4\%, e a maioria dessas mulheres tinha idade entre 30 e 39 anos, morava com companheiro, tinha renda per capita menor que meio salário mínimo e 1 a 4 anos completos de estudo, e autoavaliou sua saúde como regular.

Na Tabela 2, apresenta-se a análise univariada entre variáveis selecionadas e a realização de $\mathrm{Pa}$ -

Tabela 2. Análise univariada da realização de Papanicolaou em três ou mais anos e de nunca realização do exame em mulheres quilombolas com idade entre 18-64 anos. Projeto COMQUISTA, Bahia, Brasil, 2011.

\begin{tabular}{|c|c|c|c|c|c|c|}
\hline \multirow[t]{2}{*}{ Variáveis } & \multicolumn{3}{|c|}{ Papanicolaou $\geq 3$ anos } & \multicolumn{3}{|c|}{ Papanicolaou nunca realizado } \\
\hline & OR (IC 95\%) & $\chi^{2}$ & p - valor & OR (IC 95\%) & $\chi^{2}$ & p - valor \\
\hline \multicolumn{7}{|l|}{ Faixa etária } \\
\hline $18-24$ anos & $0.83(0.27-2.58)$ & 0.11 & 0.74 & $11.08(4.62-26.6)$ & 33.6 & $0.00^{*}$ \\
\hline $25-29$ anos & $0.87(0.30-2.55)$ & 0.06 & 0.80 & $3.18(1.21-8.32)$ & 5.79 & $0.02^{*}$ \\
\hline $30-39$ anos & $1.21(0.56-2.62)$ & 0.24 & 0.62 & $1.08(0.42-2.76)$ & 0.02 & 0.87 \\
\hline $40-49$ anos & 1.0 & & & 1.0 & & \\
\hline $50-59$ anos & $1.12(0.42-3.0)$ & 0.05 & 0.82 & $2.83(1.07-7.49)$ & 4.60 & $0.03^{*}$ \\
\hline $60-64$ anos & $1.21(0.29-5.1)$ & 0.07 & 0.79 & $2.52(0.64-9.95)$ & 1.81 & 0.18 \\
\hline \multicolumn{7}{|l|}{ Escolaridade } \\
\hline Sem instrução & $2.17(0.91-5.17)$ & 3.14 & 0.08 & $0.77(0.40-1.49)$ & 0.60 & 0.44 \\
\hline $1-4$ anos de estudo & $1.30(0.60-2.81)$ & 0.46 & 0.50 & $0.33(0.18-0.59)$ & 14.4 & $<0.01^{*}$ \\
\hline $5+$ anos de estudo & 1.0 & & & 1.0 & & \\
\hline \multicolumn{7}{|l|}{ Estado conjugal } \\
\hline Sem companheiro & $1.50(0.79-2.88)$ & 0.22 & 1.54 & $4.11(2.44-6.94)$ & 29.80 & $<0.01^{*}$ \\
\hline Com companheiro & 1.0 & & & 1.0 & & \\
\hline \multicolumn{7}{|c|}{ Autoavaliação do estado de saúde } \\
\hline Boa & $0.65(0.25-1.69)$ & 0.78 & 0.38 & $1.51(0.62-3.71)$ & 0.83 & 0.36 \\
\hline Regular & $0.64(0.25-1.61)$ & 0.91 & 0.34 & $0.88(0.35-2.19)$ & 0.07 & 0.78 \\
\hline Ruim & 1.0 & & & 1.0 & & \\
\hline \multicolumn{7}{|l|}{ Renda } \\
\hline$<1 / 2$ salário mínimo & 1.0 & & & 1.0 & & \\
\hline$\geq 1 / 2$ salário mínimo & $0.76(0.37-1.55)$ & 0.58 & 0.44 & $1.01(0.58-1.77)$ & 0.003 & 0.96 \\
\hline \multicolumn{7}{|c|}{ Realização do exame clínico das mamas } \\
\hline$<3$ anos & 1.0 & & & 1.0 & & \\
\hline$\geq 3$ anos & $4.50(1.12-18.10)$ & 5.0 & $0.02^{*}$ & $5.40(1.56-18.60)$ & 8.00 & $0.04^{*}$ \\
\hline Nunca realizou & $3.89(1.32-11.4)$ & 6.92 & $<0.01^{*}$ & $5.63(2.04-14.06)$ & 13.90 & $<0.01^{*}$ \\
\hline \multicolumn{7}{|c|}{ Consultou médico nos últimos 12 meses } \\
\hline Sim & 1.0 & & & 1.0 & & \\
\hline Não & $3.13(1.70-5.77)$ & 14.16 & $<0.01^{*}$ & $2.28(1.38-3.76)$ & 10.66 & $<0.01^{*}$ \\
\hline \multicolumn{7}{|c|}{ Procura de atendimento relacionado } \\
\hline \multicolumn{7}{|l|}{ à saúde } \\
\hline Mesmo local & 1.0 & & & 1.0 & & \\
\hline Locais diferentes & $2.91(1.44-5.90)$ & 9.30 & $<0.01^{*}$ & $2.91(1.44-5.90)$ & 16.68 & $<0.01^{*}$ \\
\hline \multicolumn{7}{|l|}{ Realização de mamografia } \\
\hline$<2$ anos & 1.0 & & & 1.0 & & \\
\hline$\geq 2$ anos & $2.83(0.27-29.96)$ & 0.80 & 0.37 & $4.72(0.50-44.70)$ & 2.11 & 0.14 \\
\hline Nunca realizada & $5.60(0.72-43.10)$ & 3.43 & 0.06 & $9.40(1.23-71.80)$ & 6.80 & $<0.01^{*}$ \\
\hline
\end{tabular}


panicolaou. Verificou-se a existência de diferenças estatisticamente significativas $(\mathrm{p}<0,05)$ entre as mulheres que realizaram Papanicolaou há três ou mais anos e as que nunca realizaram este exame em relação a: 1) faixa etária; 2) escolaridade; 3) estado conjugal; 4) realização de mamografia; 5) verificação de pressão arterial; 6) prática de atividade física; 6) realização de glicemia; 7) realização de colesterolemia/trigliceridemia. Foi observado que realizar exame clínico das mamas há três ou mais anos ou nunca realizar implicou em maior chance tanto para realização do Papanicolaou há três ou mais anos quanto para não o realizar. Observou-se uma chance de 3,13 vezes (IC 95\% 1.7 - 5.77), para realizar Papanicolaou em período maior ou igual há três anos entre as mulheres que não consultaram um médico nos últimos doze meses e uma chance 2,91 vezes (IC 95\% 1.44 - 5.90), para aquelas que buscaram atendimento relacionado à saúde em mais de uma unidade/estabelecimento de saúde.

As mulheres que não praticaram atividade física nos últimos três meses tiveram maior chance de realizar o preventivo para CCU mais tardiamente (há três ou mais anos). $\mathrm{O}$ mesmo foi observado entre aquelas que referiram nunca ter realizado glicemia e ou dosagem de colesterol/ triglicérides.

Entre as mulheres que nunca realizaram Papanicolaou foram verificadas nove características marcadoras associadas e significantes $(\mathrm{p}<0,05)$ na análise univariada. Verificaram-se maiores chances de não realização do exame entre as mulheres que não consultaram um médico nos últimos 12 meses e para as que foram buscar atendimento relacionado à saúde em locais/unidades diferentes. Também foi importante o achado de uma chance 4,11 vezes para nunca ter realizado Papanicolaou entre as mulheres sem companheiro ( IC 95\% $2.44-6.94$ ), e chances de 11,8 (IC $95 \% 4,62-26,6), 3,18$ ( IC 95\% 1,21 - 8.32) e 2,83 (IC 95\% 1.07 - 7.49) vezes respectivamente para as mulheres com idade entre 18- 24 anos, 25- 29 anos e 50-59 anos. Foi observada uma associação negativa - OR $=0,33$ ( IC $95 \% 0.18$ 0.59) entre as mulheres com 1 a 4 anos de estudo.

A análise multivariada (Tabela 3) comparou as mulheres que realizaram Papanicolaou há três anos ou mais e as que nunca realizaram o exame, com aquelas que realizaram há menos de três anos (categoria de referência). No modelo multivariado final, verificou-se que a não realização do Papanicolaou se associou significativamente à faixa etária entre 18 a 29 anos e 50 a 59 anos, não ter instrução, não ter compa- nheiro, a ter buscado atendimento relacionado à saúde em unidades/locais diferentes de seu local de residência, a ter realizado exame clínico das mamas há três ou mais anos ou nunca tê-lo realizado. Da mesma forma, realizar Papanicolaou há três anos ou mais esteve significativamente associado a não ter consultado um médico no último ano, a ter buscado atendimento à saúde em unidades/locais diferentes de seu local de residência, a nunca ter realizado glicemia ou a tê-la realizado há dois ou mais anos.

\section{Discussão}

Neste estudo, verificou-se uma prevalência de 27,3\% para não realização do exame Papanicolaou entre as mulheres quilombolas de Vitória da Conquista, que se associou de forma independente à faixa etária entre 18-29 anos e 50-59 anos, não ter nenhum nível de instrução, não ter companheiro, nunca ter feito o exame clínico das mamas, ou tê-lo executado há um tempo maior ou igual a 3 anos, e ter buscado atendimento relacionado à saúde em diferentes unidades.

Estudo realizado nas cidades de Fortaleza e Rio de Janeiro ${ }^{23}$ demonstrou que os percentuais de mulheres não submetidas ao exame de Papanicolaou foram de $19,1 \%$ e $16,5 \%$, respectivamente. Também revelou que as mulheres com baixa escolaridade, baixa renda per capita, mais idosas, solteiras, fumantes, e que nunca realizaram mamografia, exame clínico das mamas, exame de glicemia e colesterolemia, foram menos rastreadas para câncer cervical em ambas as cidades. Um recente estudo realizado em Rio Branco ${ }^{6}$ demonstrou situação semelhante para as variáveis idade, estado conjugal, renda e escolaridade. Estes resultados mostraram-se consistentes com achados de outros estudos dentro desta natureza que foram realizados em outras localidades ${ }^{24-26}$.

Os achados do presente estudo relativos à idade estão no mesmo sentido de pesquisas realizadas no Brasil dentro desta mesma linha, associando maior risco de não realização de exame preventivo para $\mathrm{CCU}$ às mulheres mais jovens (até 30 anos) e àquelas com idade superior a 50 anos, que se eximem, normalmente, porque acham desnecessária a realização deste exa$\mathrm{me}^{11,12,15,26-29}$. Além disso, para muitas mulheres, o término da idade fértil parece implicar numa diminuição da realização de consultas ginecológicas, levando ao afastamento das práticas de prevenção em um período do ciclo de vida no qual a incidência e gravidade das neoplasias são 
Tabela 3. Modelo final de regressão logística multinomial por grupo de variáveis selecionadas e realização de exame Papanicolaou. Projeto COMQUISTA, Bahia, Brasil, 2011.

\begin{tabular}{|c|c|c|c|c|}
\hline \multirow{2}{*}{ Variáveis } & \multicolumn{2}{|c|}{ Papanicolaou $\geq 3$ anos } & \multicolumn{2}{|c|}{ Papanicolaou nunca realizado } \\
\hline & OR (IC 95\%) & p - valor & OR (IC 95\%) & p - valor \\
\hline \multicolumn{5}{|l|}{ Idade } \\
\hline $18-24$ anos & $1.21(0.33-4.52)$ & $>0.05$ & $12.86(4.04-40.92)$ & $0.00^{*}$ \\
\hline $25-29$ anos & $1.18(0.36-3.90)$ & $>0.05$ & $3.59(1.11-11.56)$ & $<0.05^{*}$ \\
\hline $30-39$ anos & $1.17(0.49-2.80)$ & $>0.05$ & $1.05(0.37-2.97)$ & $>0.05$ \\
\hline $40-49$ anos & 1.0 & & 1.0 & \\
\hline $50-59$ anos & $1.60(0.52-4.88)$ & $>0.05$ & $3.56(1.13-11.19)$ & $<0.05^{*}$ \\
\hline $60-64$ anos & $0.89(0.14-5.66)$ & $>0.05$ & $2.28(0.49-10.52)$ & $>0.05$ \\
\hline \multicolumn{5}{|l|}{ Estado conjugal } \\
\hline Sem companheiro & $1.08(0.51-2.26)$ & $>0.05$ & $2.74(1.48-5.09)$ & $0.01^{*}$ \\
\hline Com companheiro & 1.0 & & 1.0 & \\
\hline \multicolumn{5}{|l|}{ Escolaridade } \\
\hline Sem instrução & $1.32(0.54-3.23)$ & $>0.05$ & $3.26(1.14-9.30)$ & $<0.05^{*}$ \\
\hline 1 - 4 anos de estudo & $2.34(0.89-6.16)$ & $>0.05$ & $2,15(0.89-5.14)$ & $>0.05$ \\
\hline $5+$ anos de estudo & 1.0 & & 1.0 & \\
\hline \multicolumn{5}{|c|}{ Consulta médica nos últimos 12 meses } \\
\hline Não & $2.14(1.08-4.25)$ & $<0.05^{*}$ & $1.57(0.84-2.92)$ & $>0.05$ \\
\hline $\operatorname{Sim}$ & 1.0 & & 1.0 & \\
\hline \multicolumn{5}{|c|}{ Local de atendimento relacionado à saúde } \\
\hline Locais diferentes & $2.61(1.17-5.79)$ & $<0.05^{*}$ & $3.37(1.58-7.17)$ & $0.001^{*}$ \\
\hline Mesmo local & 1.0 & & 1.0 & \\
\hline \multicolumn{5}{|l|}{ Exame clínico das mamas } \\
\hline$<3$ anos & 1.0 & & 1.0 & \\
\hline$\geq 3$ anos & $2.59(0.57-11.86)$ & $>0.05$ & $7.2(1.61-32.13)$ & $0.01^{*}$ \\
\hline Nunca realizou & $1.54(0.55-4.34)$ & $>0.05$ & $5.07(1.52-16.92)$ & $0.01^{*}$ \\
\hline \multicolumn{5}{|c|}{ Última dosagem de glicose sanguínea } \\
\hline$<2$ anos & 1.0 & & 1.0 & \\
\hline$\geq 2$ anos & $4.77(2.03-11.2)$ & $0.00^{*}$ & $1.43(0.62-3.33)$ & $>0.05$ \\
\hline Nunca realizou & $2.83(1.20-6.65)$ & $<0.05^{*}$ & $1.44(0.71-2.94)$ & $>0.05$ \\
\hline
\end{tabular}

*Associação estatisticamente significativa < 0.05; AIC modelo inicial = 593.81; AIC modelo final = 579.24.

mais elevadas. Não obstante essa mesma população demanda outros serviços de saúde, e poderiam ser abordadas de modo oportuno para a realização do Papanicolaou dentro de uma visão de integralidade da assistência ${ }^{11,12,15,27-30}$.

A situação conjugal tem sido relatada na literatura como um importante fator associado à não realização do exame Papanicolaou. Estudo realizado entre mulheres hispânicas identificou que a chance de não realização do exame entre as não casadas ou sem união estável era cerca de quatro vezes se comparadas às mulheres casadas $(\mathrm{OR}=4,05 \text {; IC 95\%: } 1,07-15,25)^{31}$. De modo semelhante, em um estudo realizado em Pelotas ${ }^{32}$, foi observado que ser viúva ou solteira consistia em fator de risco para não realização do exame. O presente estudo revelou que as mulheres quilombolas sem companheiro têm uma maior chance para não realização do exame Papani- colaou, o que também foi demonstrado em outros estudos nacionais ${ }^{12,26-28}$. Provavelmente, isso ocorre porque existe uma maior cobrança entre os casais em relação à busca de práticas preventivas e curativas de saúde. Outra possível explicação para esse aspecto é, que as mulheres que possuem companheiro provavelmente procuram mais os serviços de planejamento familiar ou obstétricos, oportunizando a realização do exame Papanicolaou ${ }^{33}$.

Os fatores socioeconômicos têm sido apontados como um dos elementos mais importantes relacionados ao comportamento preventivo entre as mulheres. Vários estudos mostraram associação significativa entre baixos níveis de escolaridade e renda com a ausência de rastreamento do câncer do colo do útero ${ }^{12,15,23,33-35}$.

Após ajuste final, as mulheres sem instrução apresentaram chance 3,26 vezes maior de não 
realização de exame preventivo para CCU, comparando-se com aquelas com cinco ou mais anos de escolaridade. Neste sentido, o modelo multivariado final mostrou-se consonante com resultados de outros estudos ${ }^{12,15,23,32,33,35}$ e revelou que o baixo nível de escolaridade entre as mulheres quilombolas é um importante fator de vulnerabilidade. Assim, neste contexto, a elevada prevalência de não realização do exame de Papanicolaou, é um problema a ser enfrentado com programas que garantam uma abrangência de atenção à saúde, considerando que estas mulheres experimentam inequívoca condição de desfavorecimento.

A renda não foi uma boa variável explicativa para a não realização do exame Papanicolaou. Provavelmente isso ocorreu devido à baixa renda global da população estudada. Apesar de não ter sido encontrada uma associação estatística significativa para renda, presume-se que sua baixa distribuição é um fator contributivo para a falta de equidade social no acesso ao exame de citologia oncótica. Os baixos níveis socioeconômicos têm sido associados ao desenvolvimento do câncer do colo do útero, e as populações mais vulneráveis sempre têm sido as que apresentam maiores barreiras de acesso à detecção e ao tratamento precoce, o que leva à inferência de que existe uma necessidade de maior intervenção direcionada a estes segmentos que, por sua vez, são os que mais dependem dos serviços públicos de saúde ${ }^{15}$.

Foi importante o achado de uma maior chance para realizar exame preventivo de CCU em três ou mais anos, e para não o realizar, entre as mulheres que procuraram diferentes locais para atendimento relacionado à sua saúde, comparando às que realizaram Papanicolaou em menos de 3 anos. Estes achados não diferem dos encontrados em outros estudos ${ }^{11,12,27}$, e indicam que uma possível estratégia para aumentar a cobertura do exame de Papanicolaou é incentivar um vínculo estreito de atendimento nas unidades de saúde da família (USF), onde as mulheres certamente se sentem mais familiarizadas com os profissionais, portanto mais seguras para realização de exames que exponham sua intimidade.

Esta situação pode expressar a longitudinalidade da atenção primária, que tem relevância por permitir o vínculo do usuário com a unidade e/ ou com o profissional e por estar fortemente relacionada à boa comunicação entre os envolvidos, 0 que tende a favorecer o acompanhamento do paciente, permitindo a continuidade e a efetividade de tratamentos e contribuindo também para a implementação de ações de promoção e prevenção para agravos de alta prevalência, tais como o CCU.

O modelo multivariado apontou associação estatisticamente significativa entre a não realização do exame preventivo para CCU e não realizar exame clínico das mamas em três ou mais anos ou nunca tê-lo feito, o que provavelmente se explica devido às dificuldades de acesso das mulheres quilombolas aos exames preventivos que fazem parte do rol de atendimento do programa de atenção à mulher. Também foi significativa a associação entre não consultar médico nos últimos doze meses, nunca ter feito glicemia ou tê-la realizado há dois ou mais anos com a realização do Papanicolaou em três anos ou mais. Estes achados possivelmente refletem a falta de integralidade dos serviços de saúde disponíveis para a população quilombola.

O fato de o projeto COMQUISTA ter sido um estudo baseado em informações autorreferidas implica em algumas limitações devido, principalmente, a vieses de memória, sobretudo em relação à informação do tempo de realização do exame Papanicolaou. Também o desenho transversal do estudo limitou a possibilidade de interpretar as associações de causa e efeito diretas. Por outro lado, existem pontos fortes neste estudo, dentre eles, destaca-se a obtenção pioneira de estimativas de base populacional dos fatores associados a não realização do exame Papanicolaou entre mulheres quilombolas no Município de Vitória da Conquista, que podem contribuir para o planejamento das atividades oferecidas pelo setor público à saúde da mulher.

Este estudo apresenta um alinhamento com outros trabalhos já realizados no país, e os dados encontrados indicam uma necessidade de reflexão, com o objetivo de combater fatores que se associam à não realização do Papanicolaou entre as mulheres quilombolas procurando contemplar ações para melhorar as condições de prevenção para CCU, envolvendo melhoria das condições de vida e na oferta e acesso aos serviços de saúde, sendo fundamental o envolvimento dos gestores da saúde nas três esferas, sobretudo municipal, garantindo a execução plena de ações nesta seara. 


\section{Colaboradores}

MV Oliveira, MDC Guimarães e EB França participaram igualmente de todas as etapas de elaboração do artigo.

\section{Referências}

1. Parkin DM, Whelan SL, Ferlay J, Teppo L, Thomas DB. Cancer incidence in five continents. Lyon: IARC Press; 2002. v. VIII.

2. Instituto Nacional do Câncer (Inca). Coordenação de Prevenção e Vigilância. Estimativa 2012: incidência de câncer no Brasil. Rio de Janeiro: Inca; 2011.

3. Alvarenga GC, Sá EMM, Passos MRL, Pinheiro VMS. Papilomavírus humano e carcinogênese no colo do útero. J bras Doenças Sex Transm 2000; 12(1):28-38.

4. Appleby P, , Beral V, Berrington de González A, Colin D, Franceschi S, Goodill A, Green J, Peto J, Plummer $\mathrm{M}$, Sweetland S. Carcinoma of the cervix and tobacco smoking: collaborative reanalysis of individual data on 13.541 women with carcinoma of the cervix and 23.017 women without carcinoma of the cervix from 23 epidemiological studies. Int J cancer 2006; 118(6):1481-1495.

5. Brasil. Ministério da Saúde (MS). Secretaria Nacional de Assistência à Saúde. Departamento de Atenção Básica. Cadernos de Atenção Básica no 14: Controle dos Cânceres do Colo do Útero e da Mama. Brasília: Editora do Ministério da Saúde; 2006.

6. Brasil. Ministério da Saúde. Instituto Nacional de Câncer. Câncer de Colo de Útero. [acessado 2012 jan 10]. Disponível em: http://www.inca.gov.br.

7. World Health Organization (WHO). Cancer Control Knowledge into action. WHO guide for efective pogrammes. Geneva: WHO; 2007.

8. Oliveira MMHN. Prevenção do câncer de colo uterino em São Luís: cobertura do exame de Papanicolaou e fatores associados [dissertação]. São Luís: Universidade Federal do Maranhão; 1999.

9. Oliveira AF, Cunha CLF, Viégas IV, Figueiredo IS, Brito LMO, Chein MBC. Estudo sobre a adesão ao exame citopatológico de Papanicolau em um grupo de mulheres. Rev Pesquisa em Saúde 2011; 11(1):32-37.

10. Arbyn M, Dillner J. European Guidelines for Quality Assurance in Cervical Cancer Screening. Second edition summary document. Ann Oncol 2010; 21(3):448458.

11. Dias-da-Costa JS, Olinto MTA, Gigante DP, Menezes AMB, Macedo S, Borba AT, Motta GLS, Fuchs SC. Cobertura do exame citopatológico na cidade de Pelotas, Rio Grande do Sul, Brasil. Cad Saude Publica 2003; 19(1):191-197.

12. Martins LFL, Thuler LCS, Valente JG. Cobertura do exame de Papanicolaou no Brasil e seus fatores determinantes: uma revisão sistemática da literatura. Rev. Bras. Ginecol. Obstet. 2005; 27(8):485-492.

13. World Health Organization (WHO). Women and Cervical and Breast Cancer. [acessado 2012 mar 3]. Disponível em: http://www.who.int.

14. Thuler LCS. Lesões precursoras do câncer do colo uterino - Aspectos epidemiológicos. Figueiredo EMA, Moralez GM, organizadores. Ginecologia oncológica. Rio de Janeiro: Revinter; 2004. p. 3-6.

15. Amorim VMSL, Barros MBA, César CLG, Carandina L, Goldbaum M. Fatores associados a não realização do exame de Papanicolaou: um estudo de base populacional no Município de Campinas, São Paulo, Brasil. Cad Saude Publica 2006; 22(11):2329-2338. 
16. Lopes F. Experiências desiguais ao nascer, viver, adoecer e morrer: tópicos em saúde da população negra no Brasil. In: Brasil. Fundação Nacional de Saúde. Saúde da população negra no Brasil: contribuições para a promoção da equidade. Brasília: Funasa; 2005. p. 446.

17. Coimbra CEA, Santos RV. Saúde, minorias e desigualdade: algumas teias de inter-relações, com ênfase nos povos indígenas no Brasil. Cien Saude Colet 2000; 5(1):125-132.

18. Comissão Nacional sobre Determinantes Sociais da Saúde (CNDSS). As Causas Sociais das iniquidades da saúde no Brasil. Rio de Janeiro: Ed. Fiocruz; 2008.

19. Projeto COMQUISTA. Comunidades Quilombolas de Vitória da Conquista - BA: Avaliação de Saúde e seus Condicionantes. [acessado 2014 set 3]. Disponível em: http://projetocomquista.wordpress.com

20. Brasil. Datasus. Cadastro nacional de estabelecimentos de saúde. [acessado 2014 set 3]. Disponível em: http:// cnes.datasus.gov.br/Index.asp?home $=1$

21. Fiocruz. Projeto de planejamento da Pesquisa nacional de saúde. [acessado 2014 set 3]. Disponível em: http:// www.pns.icict.fiocruz.br/

22. Bezerra VM, Medeiros DS, Gomes KO, Souzas R, Giatti L, Steffens AP, Kochergin CN, Souza CL, Moura CS, Soares DA, Santos LRCS, Cardoso LGV, Oliveira MV, Martins PC, Neves OSC, Guimarães MDC. Inquérito de Saúde em Comunidades Quilombolas de Vitória da Conquista, Bahia, Brasil (Projeto COMQUISTA): aspectos metodológicos e análise descritiva. Cien Saude Colet 2014; 19(6):1835-1847.

23. Martins LFL, Valente JG, Thuler LCS. Fatores associados a não realização do exame de Papanicolaou: estudo transversal de base populacional em duas capitais brasileiras. Rev Bras Cancerol 2006; 52(2):197.

24. Muller DK, Dias-da-Costa JS, Luz AMH, Olinto MTA. Cobertura do exame citopatológico do colo do útero na cidade de São Leopoldo, Rio Grande do Sul, Brasil. Cad Saude Publica 2008; 24(11):2511-2520.

25. Carlotto K, Cesar JA, Hackenhaar AA, Ribeiro PRP. Características reprodutivas e utilização de serviços preventivos em saúde por mulheres em idade fértil: resultados de dois estudos transversais de base populacional no extremo Sul do Brasil. Cad Saude Publica 2008; 24(9):2054-2062.

26. Albuquerque KM, Frias PG, Andrade CLT, Aquino EMC, Menezes G, Szwarcwald CL. Cobertura do teste de Papanicolaou e fatores associados a não realização: um olhar sobre o Programa de Prevenção do Câncer do Colo do Útero em Pernambuco, Brasil. Cad Saude Publica 2009; 25(Supl. 2):S3019.
27. Nascimento CM, Eluf-Neto J, Rego RA. Pap test coverage in São Paulo municipality and characteristics of the women tested. Bull Pan Am Health Organ 1996; 30(4):302-312.

28. Cesar JA, Horta BL, Gomes G, Houlthausen RS, Willrich RM, Kaercher A, Kaercher A, Iastrenski FM. Fatores associados a não realização de exame citopatológico de colo uterino no extremo Sul do Brasil. Cad Saude Publica 2003; 19(5):1365-1372.

29. Gasperin SI, Boing AF, Kupek E. Cobertura e fatores associados à realização do exame de detecção do câncer de colo de útero em área urbana no Sul do Brasil: estudo de base populacional. Cad Saude Publica 2011; 27(7):1312-1322.

30. Zeferino LC, Pinotti JA, Jorge JPN, Westin MCA, Tambascia JK, Montemor EBL. Organization of cervical cancer screening in Campinas and surrounding region, São Paulo State, Brazil. Cad Saude Publica 2006; 22(9):1909-1914.

31. Koval AE, Riganti AA, Foley KL. CAPRELA (Cancer Prevention for Latinas): findings of a pilot study in Winston-Salem, Forsyth County. N C Med J 2006; 67(1):9-16.

32. Quadros CAT, Victora CG, Dias-da-Costa JS. Coverage and focus of a cervical cancer prevention program in southern Brazil. Rev Panam Salud Publica 2004; 16(4):223-232.

33. McPhee SJ, Nguyen TT. Cancer, cancer risk factors, and community based cancer control trials in Vietnamese Americans. Asian Am Pac Isl J Health 2000; 8(1):18-31.

34. Borges MFSO, Dotto LMG, Koifman RJ, Cunha MA, Muniz PT. Prevalência do exame preventivo de câncer do colo do útero em Rio Branco, Acre, Brasil, e fatores associados a não-realização do exame. Cad Saude Publica 2012; 28(6):1156-1166.

35. Silva DW, Andrade SM, Soares DA, Tutini B, Schneck CA, Lopes ML. Cobertura e fatores associados com a realização do exame Papanicolaou em município do Sul do Brasil. Rev Bras Ginecol Obstet 2006; 28(1):2431.

Artigo apresentado em 31/07/2013

Aprovado em 27/08/2013

Versão final apresentada em 31/08/2013 